\title{
Lactotripeptides effect on office and 24-h ambulatory blood pressure, blood pressure stress response, pulse wave velocity and cardiac output in patients with high-normal blood pressure or first-degree hypertension: a randomized double-blind clinical trial
}

\author{
Arrigo FG Cicero, Martina Rosticci, Beatrice Gerocarni, Stefano Bacchelli, Maddalena Veronesi, \\ Enrico Strocchi and Claudio Borghi
}

Contrasting data partially support a certain antihypertensive efficacy of lactotripeptides (LTPs) derived from enzymatic treatment of casein hydrolysate. Our aim was to evaluate this effect on a large number of hemodynamic parameters. We conducted a prospective double-blind randomized clinical trial, which included 52 patients affected by high-normal blood pressure (BP) or first-degree hypertension. We investigated the effect of a 6-week treatment with the LTPs isoleucine-proline-proline and valineproline-proline at $3 \mathrm{mg}$ per day, assumed to be functional food, on office BP, 24-h ambulatory BP monitoring (ABPM) values, stress-induced BP increase and cardiac output-related parameters. In the LTP-treated subjects, we observed a significant reduction in office systolic $\mathrm{BP}(\mathrm{SBP} ;-5 \pm 8 \mathrm{~mm} \mathrm{Hg}, P=0.013)$ and a significant improvement in pulse wave velocity (PWV; $-0.66 \pm 0.81 \mathrm{~m} \mathrm{~s}^{-1}, P=0.001$; an instrumental biomarker of vascular rigidity). No effect on 24-h ABPM parameters and BP reaction to stress was observed from treatment with the combined LTPs. LTPs, but not placebo, were associated with a mild but significant change in the stroke volume (SV), SV index (markers of cardiac flow), the acceleration index (ACI) and velocity index (VI) (markers of cardiac contractility). No effect was observed on parameters related to fluid dynamics or vascular resistance. LTPs positively influenced the office SBP, PWV, SV, SV index, ACI and VI in patients with high-normal BP or first-degree hypertension.

Hypertension Research (2011) 34, 1035-1040; doi:10.1038/hr.2011.92; published online 14 July 2011

Keywords: blood pressure; cardiac output; lactotripeptides; pulse wave velocity

\section{INTRODUCTION}

Beyond the well-known effects on blood pressure (BP) of the Dietary Approaches to Stop Hypertension ${ }^{1}$ plan and the Mediterranean diets, ${ }^{2}$ a recent meta-analysis has suggested that some peptides derived from food proteins may lead to a significant reduction of systolic BP (SBP: $-5.13 \mathrm{~mm} \mathrm{Hg} ; 95 \%$ confidence intervals (CI): $-7.1 /-3.1$ ) and diastolic BP (DBP: $-2.42 \mathrm{~mm} \mathrm{Hg}$; 95\% CI: $-3.8 /-1.0){ }^{3}$ In particular, these peptides that are part of the primary structure of milk proteins and can be released by enzymatic hydrolysis, either during gastrointestinal digestion or during food processing, have been reported to exert some angiotensin-converting enzyme inhibitory activity. ${ }^{4}$ A detailed description of the individual milk proteins with in vitro angiotensin-converting enzyme-inhibitory activity is beyond the purpose of this paper and has been reviewed elsewhere. ${ }^{5}$ However, the best characterized peptides are those found in fermented milk that contain the amino acid tripeptide sequence isoleucine-proline-proline (IPP) and valine-proline-proline (VPP). For the most part, researchers have concentrated their efforts on finding a short-term antihypertensive effect of these peptides on office-measured BP. A recent meta-analysis by our group ${ }^{6}$ showed that the pooled effect of peptides gave a reduction of $-3.73 \mathrm{~mm} \mathrm{Hg}(95 \% \mathrm{CI}:-6.70,-1.76)$ for SBP and $1.97 \mathrm{~mm} \mathrm{Hg}(95 \% \mathrm{CI}:-3.85,-0.64)$ for DBP. The effect was more evident in Asian patients ( $\mathrm{SBP}=-6.93 \mathrm{~mm} \mathrm{Hg}(95 \%$ CI: $-10.95,-2.94) ; \mathrm{DBP}=-3.98 \mathrm{~mm} \mathrm{Hg}(95 \% \mathrm{CI}:-5.38,-2.44)$ ) than in Caucasians ( $\mathrm{SBP}=-1.17 \mathrm{~mm} \mathrm{Hg}$ (95\% CI: $-2.82,0.72)$; $\mathrm{DBP}=-0.52 \mathrm{~mm} \mathrm{Hg}(95 \% \mathrm{CI}:-1.39,0.13))$ and apparently was not related to age, baseline BP values, assumed dose of lactotripeptides (LTPs) or length of the treatment. There are only a few studies that have extended the clinical investigation to the antihypertensive activity of LTPs, including 24-h ambulatory BP monitoring (ABPM) values, 
and contrasting results have been obtained. A positive effect was observed in the US, ${ }^{7}$ Japanese $^{8}$ and southern European subjects, ${ }^{9}$ but not in northern Europeans. ${ }^{10}$ In Japanese subjects only, investigations confirmed an effect of LTP on flow-mediated dilation, ${ }^{11}$ artery compliance, ${ }^{12}$ and central BP. ${ }^{13}$ Because the antihypertensive effect of LTPs seems to be strongly related to the ethnicity of patients, ${ }^{6}$ it would be beneficial to increase our understanding of the effect of these nutraceuticals on different cardiovascular parameters in European subjects.

In this context, the aim of our study was to evaluate the effect of LTPs supplemented as functional food on a large number of hemodynamic parameters in southern European subjects with high-normal $\mathrm{BP}$ or first-degree hypertension.

\section{METHODS}

The study was conducted in a group of volunteers with normal or high-normal $\mathrm{BP}$ according to a randomized double-blind design. We consecutively enrolled 50 adult untreated subjects (men:women=29:21) aged 25-70 years old $(51.2 \pm 10.94$ years old $)$ with high-normal $\mathrm{BP} \quad(\mathrm{SBP}=130-139 \mathrm{~mm} \mathrm{Hg}$; $\mathrm{DBP}=85-89 \mathrm{~mm} \mathrm{Hg}$ ) or first-degree hypertension $(\mathrm{SBP}=140-159 \mathrm{~mm} \mathrm{Hg}$; $\mathrm{DBP}=90-99 \mathrm{~mm} \mathrm{Hg}$ ) as defined previously ${ }^{14}$ and in primary prevention for cardiovascular disease. This was the patients' first visit to the Hypertension Clinic of the Department of Internal Medicine, Aging and Kidney Disease, University of Bologna, Bologna, Italy.

Patients with extreme variability of BP, as observed by patient diaries $( \pm 10 \%)$ in the month before the beginning of the study, receiving antihypertensive medications, those with chronic gastrointestinal disorders and receiving medication for the treatment of these disorders, with kidney function abnormalities and/or known intolerance to the component of the tested products were excluded from the study.

All patients were evaluated at baseline where they were given standard behavioral and dietary suggestions to correct unhealthy habits. After 4 weeks, they were allocated to treatment with fruit juice containing $3 \mathrm{mg}$ of added IPP/ VPP LTPs from casein (AmealPeptide, kindly furnished by Calpis, Tokyo, Japan) or with placebo (kindly furnished by Barilla SpA, Parma, Italy). Randomization was performed by using a drawing of envelopes that contained randomization codes prepared by an independent statistician and specific software. The envelopes were then further mixed and distributed to the investigators who assigned the randomization code in a progressive way to the enrolled subjects. A copy of the code was provided only to the person responsible for performing the statistical analysis. The juice bottles were indistinguishable by color and taste. The patients were instructed to take one bottle $(250 \mathrm{ml})$ in the morning with breakfast. Each serving provided $90 \mathrm{kcal}$ $(383 \mathrm{~kJ}), 1 \mathrm{mg}$ of IPP and $2 \mathrm{mg}$ of VPP. After 6 weeks of treatment, the patients were given a final evaluation to determine the treatment efficacy. Product compliance was assessed by counting the number of product doses returned at the time of the final study visit.

The study was conducted in accordance with the Declaration of Helsinki. The study protocol was approved by the Ethics Committee of the University of Bologna, and informed consent was obtained from all patients before inclusion in the study.

\section{Blood pressure measurement}

BP measurements were taken from each patient (using the right arm) in the seated position using a standard mercury sphygmomanometer (Erkameter 3000, ERKA, Bad Tolz, Germany; Korotkoff I and V) with a cuff of appropriate size. BP was measured by the same investigator at each visit in the morning before daily drug intake and after the patient had rested for $10 \mathrm{~min}$ in a quiet room. Three successive BP readings were obtained at 1-min intervals, and the mean of the three readings was calculated. The BP measurements were performed by physicians not belonging to the study to preserve the study blinding. ${ }^{15}$

ABPM was performed for $24 \mathrm{~h}$, using a non-invasive automatic monitor (Space Labs, Redmond, WA, USA, model 90207). For ABPM, patients were instructed to act and work normally between $0600 \mathrm{~h}$ and $2200 \mathrm{~h}$ and rest or sleep between $2200 \mathrm{~h}$ and $0600 \mathrm{~h}$. They were not to receive any antihypertensive drug treatment at any time, nor any other type of drug that might affect their BP level. Readings were obtained automatically at 15-min intervals throughout a 24-h study period. Separate averages were obtained for the 24-h, daytime $(0600 \mathrm{~h}-2200 \mathrm{~h})$ and night time $(2200 \mathrm{~h}-0600 \mathrm{~h})$ values. The accuracy of the automatic BP readings was checked twice for each ABPM against manual readings, taken using a standard mercury sphygmomanometer. BP was measured with the patient sitting, before the beginning of the ABPM, and after a 5 -min rest period. Three readings were obtained and averaged. The accuracy test was repeated after the end of each 24-h ABPM. Patients with more than a 5 - $\mathrm{mm} \mathrm{Hg}$ difference in SBP between the manual and automatic readings were excluded from further analysis. All sampled records were decoded by a trained physician in a double-blind manner. ${ }^{16}$

\section{Handgrip stressor test}

Short-term moderate static handgrip exercise $(30 \%)$ elicits an increase in multiunit post-ganglionic sympathetic nerve activity, directed to muscles by activating metaboreceptors, mechanoreceptors and central command. The participants were asked to squeeze a hand dynamometer (Stoelting, Wood Dale, IL, USA) with maximal force. The highest force attained from three attempts was considered the maximum voluntary contraction (MVC). Static handgrip exercise $30 \%$ was performed by squeezing the hand dynamometer at $30 \%$ of maximum voluntary contraction in a sustained manner for $3 \mathrm{~min}$. The participant was instructed to avoid performing an inadvertent Valsalva maneuver and to maintain normal breathing patterns. BP was measured at the baseline after the sustained squeezing phase and after a 3 -min rest. ${ }^{17}$

\section{Pulse wave measurement}

The pulse wave velocity (PWV) was calculated using the SphygmoCor System (Vx Pulse Wave Velocity; AtCor Medical Head Office, West Ryde, Australia), with the patient in the supine decubitus position. The carotid and femoral pulse wave were analyzed, the delay in the electrocardiogram wave was estimated and the PWV was calculated. Using a measuring tape, the space measurements were taken from the suprasternal notch to the carotid and femoral arteries at the sensor location. ${ }^{18}$

\section{Cardiac output evaluation}

After rubbing and cleaning the skin with alcohol to achieve a skin-to-electrode impedance as low as possible, two sensors including gel pads were carefully placed on each side of the thorax along the midaxillary line, and the two remaining sensors were placed on each side of the neck just above the clavicle. An alternating current of $1.5 \mathrm{~mA}(85 \mathrm{kHz})$ was applied and the impedance cardiographic signal was continuously displayed on the screen, together with the electrocardiographic signal. The impedance cardiographic signal was the first derivative of the thoracic impedance $(\mathrm{d} Z / \mathrm{d} t)$. Bioimpedance values were updated on the impedance cardiographic monitor every 15 heartbeats. An impedance cardiographic quality indicator, which represented the percentage of the evaluable heartbeats, was determined. Distortions or artifacts in the impedance cardiographic signal were detected and excluded from the analysis. In case of excessive distortions or artifacts, the test was repeated to obtain an acceptable report. With impedance cardiography, the left ventricular ejection time (LVET) was directly measured. The left ventricular stroke volume (SV) was calculated using the formula $V_{\mathrm{EPT}} \times \mathrm{d} Z_{\max } / Z_{0} \times \mathrm{LVET}$, where $V_{\mathrm{EPT}}$ was calculated from the patient's data (weight, height, age, gender), $Z_{\max }$ represented the amplitude of the systolic wave of the impedance cardiography and $Z_{0}$ the base impedance. The SV calculation was computed every single heartbeat and averaged over 15 heartbeats to account for physiological respiratory variations of the SV. The cardiac index was then calculated based on the SV, the heart rate and the body surface area. The thoracic fluid content, an expression of thoracic fluid level that could be useful for evaluating thoracic blood volume, was calculated as $1000 / Z_{0}$, where $Z_{0}$ indicated the base impedance (Sonosite, Hitchin, Herts, GB, UK). Raw data were analyzed offline and all impedance cardiographic measurements were performed with a single monitor using PC-version 1.9 of the Niccomo software. ${ }^{19}$ 


\section{Laboratory data}

All plasma parameters were obtained after a 12-h overnight fast. Venous blood samples were drawn by a nurse in all patients between $0800 \mathrm{~h}$ and $0900 \mathrm{~h}$. Plasma was obtained by addition of $\mathrm{Na}_{2}-\mathrm{EDTA}, 1 \mathrm{mg} \mathrm{ml}^{-1}$ and centrifuged at $3000 \mathrm{~g}$ for $15 \mathrm{~min}$ at $4^{\circ} \mathrm{C}$. Immediately after centrifugation, the plasma samples were frozen and stored at $-80^{\circ} \mathrm{C}$ for no more than 3 months. The following parameters were evaluated: total cholesterol, high-density lipoprotein cholesterol, triglycerides, glucose, uric acid, liver transaminases and creatinine via standardized methods. All measurements were centrally performed in the laboratory of our department. Laboratory technicians drew the blood samples and a biologist performed the assays using standardized methods. ${ }^{20}$

\section{Statistical analysis}

An intention-to-treat analysis was conducted in the patients who had received $\geqslant 1$ dose of study medication and had a subsequent efficacy observation. The distribution normality of the tested parameters was evaluated by the Kolmogorov-Smirnov test. Continuous variables were compared by analysis of variance. Intervention effects were adjusted for additional potential confounders using analysis of covariance. Analysis of variance was also used to assess the significance within and between groups. Outcome variables with a skewed distribution were transformed to a log scale before statistical testing. The statistical significance of the independent effects of treatments on the other variables was determined using analysis of covariance. A one-sample $t$-test was used to compare values obtained before and after treatment administration; two-sample $t$-tests were used for between-group comparisons. The Bonferroni correction for multiple comparisons was also carried out. All analyses were repeated by further sub-dividing patients for those with high-normal BP and first-degree hypertension. All data were expressed as mean and s.d. A $P$-level less than 0.05 was considered significant for all tests.

\section{RESULTS}

Fifty-four patients were screened and four screening failures occurred. At the screening visit, the main characteristics of the enrolled patients (25 with high-normal BP, 25 with first-degree hypertension) were: body mass index $=26.7 \pm 3.7 \mathrm{~kg} \mathrm{~m}^{-2}, \mathrm{SBP}=138.8 \pm 8.8 \mathrm{~mm} \mathrm{Hg}$, $\mathrm{DBP}=87.7 \pm 6.0 \mathrm{mmHg}$, pulse pressure $=51.1 \pm 10.5 \mathrm{~mm} \mathrm{Hg}$, and heart rate $=71.1 \pm 10.2$ b.p.m.

Only two patients withdrew from the study because of personal problems (no safety issues were encountered).

Basic hemodynamic and laboratory data at the baseline in subjects randomized to LTPs or placebo treatment are presented in Table 1. The treatment groups were cross-matched for baseline age, body mass index, waist circumference, BP and PWV. No significant changes were observed in the placebo group during the study, whereas in the LTP-treated patients, SBP $(-4.8 \pm 8.7 \mathrm{~mm} \mathrm{Hg}, P=0.013)$ and PWV $\left(-0.66 \pm 0.80 \mathrm{~m} \mathrm{~s}^{-1}\right)$ significantly improved when compared with the baseline (Figure 1). The PWV change was also significantly higher compared with that observed in the placebo group. The PWV change was significantly related to its baseline value in LTP-treated patients $(\beta=-0.518, P=0.010)$, but not in those taking placebo $(\beta=-0.375$, $P=0.710$ ) (Figure 2). No significant relationship was observed between SBP and PWV change $(\beta=-0.003, P=0.843)$. The other basic variables, including those from the laboratory, did not change in either the LTP or the placebo group.

No statistically significant differences between LTP- and placebotreated subjects, either in those with high-normal hypertension or with first-degree hypertension at baseline, were observed for the ABPM data (Table 2) and the handgrip stress test (Table 3). Among cardiac output-related parameters, SV $(P=0.013)$, stroke index $(P=0.049)$, the acceleration index $(\mathrm{ACI} ; P=0.044)$ and velocity index (VI; $P=0.048$ ) significantly improved, compared with the baseline in the LTP-treated subjects only (Table 4). No effect was observed on parameters related to fluid dynamics or vascular
Table 1 Basic hemodynamic and laboratory data at the baseline in subjects randomized to lactotripeptides or placebo treatment

\begin{tabular}{|c|c|c|c|c|}
\hline & \multicolumn{2}{|c|}{ Lactotripeptides (N. 25) } & \multicolumn{2}{|c|}{ Placebo (N. 25) } \\
\hline & Mean & s.d. & Mean & s.d. \\
\hline WC $(\mathrm{cm})$ & 98.16 & 8.26 & 97.62 & 12.54 \\
\hline $\mathrm{BMI}\left(\mathrm{kg} \mathrm{m}^{-2}\right)$ & 26.61 & 3.23 & 27.01 & 4.07 \\
\hline $\mathrm{SBP}(\mathrm{mm} \mathrm{Hg})$ & 141.96 & 10.88 & 141.20 & 9.64 \\
\hline $\mathrm{DBP}(\mathrm{mm} \mathrm{Hg})$ & 86.36 & 8.22 & 86.62 & 7.72 \\
\hline $\mathrm{PP}(\mathrm{mm} \mathrm{Hg})$ & 55.60 & 11.12 & 54.58 & 8.53 \\
\hline HR (b.p.m.) & 71.68 & 9.59 & 70.54 & 10.38 \\
\hline $\mathrm{TC}\left(\mathrm{mg} \mathrm{dl}^{-1}\right)$ & 211.96 & 34.09 & 217.04 & 31.27 \\
\hline $\mathrm{TG}\left(\mathrm{mg} \mathrm{dl} \mathrm{l}^{-1}\right)$ & 121.96 & 67.20 & 113.44 & 43.53 \\
\hline HDL-C (mg dl-1 $\left.{ }^{-1}\right)$ & 54.77 & 11.90 & 58.20 & 13.58 \\
\hline LDL-C (mg dl $\left.{ }^{-1}\right)$ & 132.80 & 27.29 & 136.15 & 30.54 \\
\hline $\mathrm{FPG}\left(\mathrm{mg} \mathrm{dl}^{-1}\right)$ & 86.96 & 9.86 & 86.96 & 11.26 \\
\hline Creatinine $\left(\mathrm{mg} \mathrm{dl}^{-1}\right)$ & 0.89 & 0.15 & 0.91 & 0.12 \\
\hline SUA (mg dl-1) & 5.54 & 1.19 & 5.58 & 1.07 \\
\hline GOT (mg dl-1) & 20.57 & 4.30 & 22.72 & 6.99 \\
\hline GPT (mg dl-1) & 23.69 & 10.30 & 26.52 & 11.58 \\
\hline
\end{tabular}

Abbreviations: BMI, body mass index; DBP, diastolic blood pressure; FPG, fasting plasma glucose; GOT, glutamic-oxalacetate transaminase; GPT, glutamic-pyruvate transaminase HDL-C, high density lipoprotein cholesterol; LDL-C, low density lipoprotein cholesterol; PP, pulse pressure; SBP, systolic blood pressure; SUA, serum uric acid; TC, total cholesterol; TG, triglycerides; WC, waist circumference.
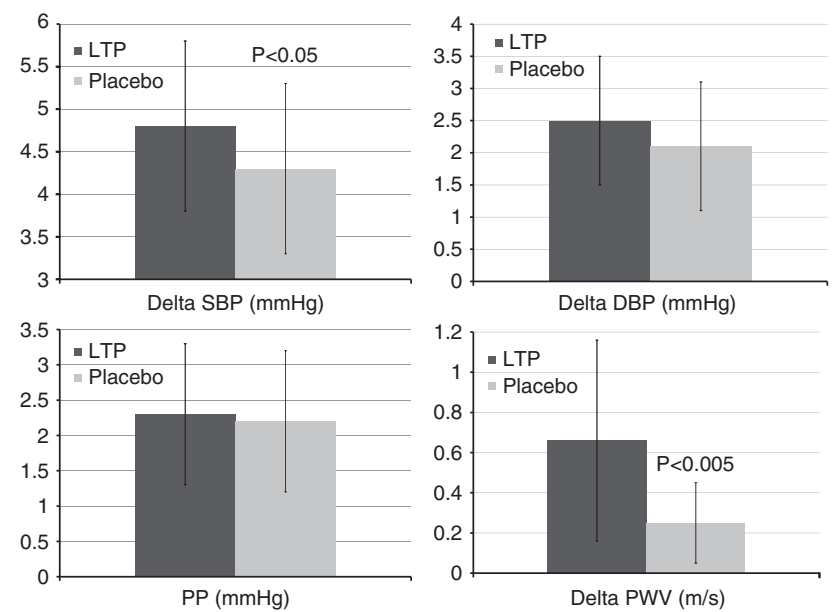

Figure 1 Changes in blood pressure (BP) and pulse wave velocity (PWV) observed during the study in the lactotripeptides (LTP) and placebo-treated patients.

resistance. Overall, no differences were observed between subjects with high-normal hypertension or first-degree hypertension at baseline.

\section{DISCUSSION}

On one hand, a large body of evidence suggests a certain antihypertensive efficacy of LTPs, ${ }^{6}$ whereas on the other hand, recent studies do not support the presumed angiotensin-converting enzyme inhibitorlike activity of these peptides. ${ }^{21}$ In this context, we conducted a clinical trial and included advanced tests to better understand the possible efficacy of these peptides and their mechanism of action. 
Beyond the small but significant effect of reducing SBP, the limited antihypertensive efficacy of LTPs on office-measured BP is in agreement with the results recently reported in northern European subjects by van der Zanker et al. ${ }^{22}$ Even if the observed effect on SBP is negligible, the reduction of DBP could have some potential prognostic value, considering that the enrolled patients were all relatively young. Recently, it has also been proposed that the mild peripheral antihypertensive effect of LTPs is associated with improvements in central arterial stiffness. ${ }^{13}$

The lack of observations of an LTP effect on ABPM data is partially in contrast with our data previously obtained on normotensive

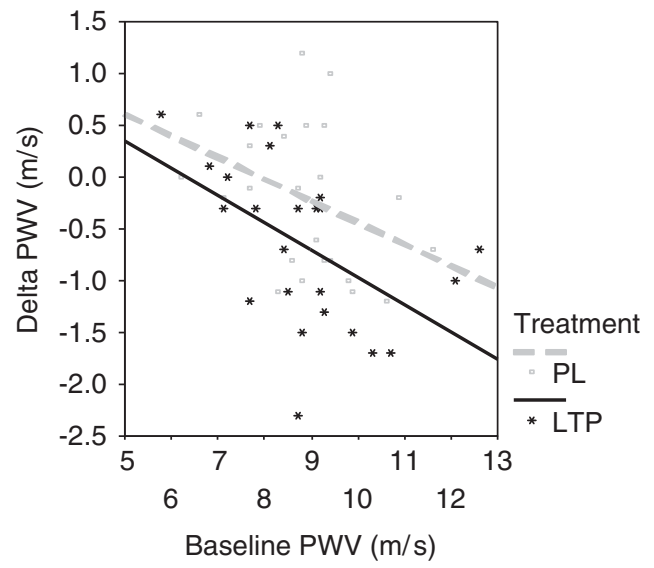

Figure 2 Pulse wave velocity (PWV) changes and baseline PWV in placeboand lactotripeptide (LTP)-treated patients. subjects and patients with high-normal BP. ${ }^{9}$ This may be because we administered the functional food twice daily in that study, whereas in this study, we administered it once daily and the halflives of the LTPs are short, presumably. Similarly, a non-significant LTP effect on BP has also been observed in other European studies where the product was administered once a day. ${ }^{10,23}$ In contrast, when the product was administered twice daily, a significant improvement in ABPM parameters was observed. ${ }^{24}$

What is new in our study is the evaluation of a large number of surrogate parameters of cardiovascular risk. Increased arterial stiffness, which is assessed by the PWV, causes increased cardiac afterload,

Table 3 Changes in blood pressure level from baseline to after the handgrip stress, and from the end of the stress to the resting phase in lactrotripeptides- and placebo-treated patients (values reported as mean \pm s.d.)

\begin{tabular}{|c|c|c|c|c|}
\hline & \multicolumn{2}{|c|}{ Placebo } & \multicolumn{2}{|c|}{ Lactotripeptides } \\
\hline & After stress & After recover & After stress & After recover \\
\hline \multicolumn{5}{|c|}{ Pre-treatment values } \\
\hline $\mathrm{SBP}(\mathrm{mm} \mathrm{Hg})$ & $17.0 \pm 12.8$ & $-18.2 \pm 13.4$ & $15.5 \pm 11.0$ & $-16.39 \pm 10.1$ \\
\hline $\mathrm{DBP}(\mathrm{mm} \mathrm{Hg})$ & $10.7 \pm 8.1$ & $-9.8 \pm 11.1$ & $7.5 \pm 9.8$ & $-8.3 \pm 5.8$ \\
\hline \multicolumn{5}{|c|}{ Post-treatment values } \\
\hline $\mathrm{SBP}(\mathrm{mm} \mathrm{Hg})$ & $17.8 \pm 11.9$ & $-16.8 \pm 15.3$ & $15.5 \pm 11.4$ & $-17.3 \pm 11.9$ \\
\hline $\mathrm{DBP}(\mathrm{mm} \mathrm{Hg})$ & $8.9 \pm 7.6$ & $-8.4 \pm 9.6$ & $10.9 \pm 7.3$ & $-9.2 \pm 9.2$ \\
\hline
\end{tabular}

Abbreviations: DBP, diastolic blood pressure; SBP, systolic blood pressure.

Table 2 24-h ambulatory blood pressure measurement parameters during the treatment with lactotripeptides or placebo (values reported as mean \pm s.d.)

\begin{tabular}{|c|c|c|c|c|}
\hline & \multicolumn{2}{|c|}{ Placebo } & \multicolumn{2}{|c|}{ Lactotripeptides } \\
\hline & Basal & 6 week & Basal & 6 weeks \\
\hline $\mathrm{DBP}(\mathrm{mm} \mathrm{Hg})$ & $83.4 \pm 5.5$ & $82.5 \pm 7.4$ & $82.6 \pm 8.5$ & $82.6 \pm 8.2$ \\
\hline $\mathrm{MBP}(\mathrm{mm} \mathrm{Hg})$ & $100.1 \pm 5.6$ & $98.6 \pm 7.6$ & $99.3 \pm 8.6$ & $98.9 \pm 8.0$ \\
\hline $\mathrm{PP}(\mathrm{mm} \mathrm{Hg})$ & $49.5 \pm 6.6$ & $48.6 \pm 7.1$ & $49.4 \pm 5.8$ & $49.3 \pm 6.2$ \\
\hline$\% \mathrm{DBP}>$ normal & $52.4 \pm 20.2$ & $50.2 \pm 24.0$ & $48.2 \pm 28.5$ & $49.7 \pm 28.2$ \\
\hline Diurnal SBP $(\mathrm{mm} \mathrm{Hg})$ & $137.6 \pm 9.2$ & $136.1 \pm 10.6$ & $136.7 \pm 9.7$ & $135.6 \pm 8.5$ \\
\hline Diurnal DBP (mm Hg) & $87.8 \pm 6.2$ & $86.9 \pm 6.7$ & $87.2 \pm 9.2$ & $86.6 \pm 9.2$ \\
\hline Diurnal MBP $(\mathrm{mm} \mathrm{Hg})$ & $104.5 \pm 6.8$ & $103.0 \pm 7.2$ & $103.9 \pm 9.2$ & $102.8 \pm 8.4$ \\
\hline Diurnal PP (mm Hg) & $49.9 \pm 7.1$ & $49.2 \pm 8.1$ & $49.5 \pm 6.0$ & $49.0 \pm 6.7$ \\
\hline Diurnal HR (p.p.m.) & $75.2 \pm 8.1$ & $76.1 \pm 9.9$ & $76.0 \pm 9.6$ & $75.7 \pm 10.2$ \\
\hline Night MBP $(\mathrm{mm} \mathrm{Hg})$ & $88.3 \pm 9.2$ & $86.7 \pm 8.9$ & $90.2 \pm 8.9$ & $91.3 \pm 9.4$ \\
\hline Night PP (mm Hg) & $49.2 \pm 5.9$ & $47.7 \pm 6.4$ & $49.2 \pm 6.6$ & $48.1 \pm 11.1$ \\
\hline Night HR (p.p.m.) & $65.6 \pm 7.4$ & $66.0 \pm 8.4$ & $64.5 \pm 8.5$ & $66.5 \pm 6.8$ \\
\hline$\%$ Night SBP $>$ normal & $56.5 \pm 24.3$ & $45.7 \pm 31.1$ & $52.5 \pm 30.4$ & $56.7 \pm 30.7$ \\
\hline$\%$ Night $\mathrm{DBP}>$ normal & $36.4 \pm 24.4$ & $31.8 \pm 28.9$ & $29.3 \pm 28.2$ & $40.9 \pm 37.3$ \\
\hline
\end{tabular}

Abbreviations: DBP, diastolic blood pressure; HR, heart rate; MBP, mean blood pressure; PP, pulse pressure; SBP, systolic blood pressure. 
Table 4 Cardiac output-related parameters before and after treatment with lactotripeptides or placebo (values reported as mean \pm s.d.)

\begin{tabular}{|c|c|c|c|c|}
\hline & \multicolumn{2}{|c|}{ Placebo } & \multicolumn{2}{|c|}{ Lactotripeptides } \\
\hline & Basal & 6 week & Basal & 6 weeks \\
\hline $\mathrm{CO}\left(I \mathrm{~min}^{-1}\right)$ & $5.1 \pm 1.2$ & $4.9 \pm 1.3$ & $5.2 \pm 1.2$ & $5.0 \pm 1.2$ \\
\hline $\mathrm{Cl}\left(\mathrm{Imin}^{-1} \mathrm{~m}^{-2}\right)$ & $2.4 \pm 0.7$ & $2.4 \pm 0.5$ & $2.7 \pm 0.5$ & $2.6 \pm 0.5$ \\
\hline SVR (dyne sec $\mathrm{cm}^{-5}$ ) & $1720.4 \pm 480.7$ & $1716.8 \pm 555.8$ & $1652.9 \pm 416.9$ & $1706.7 \pm 473.8$ \\
\hline $\mathrm{SI}\left(\mathrm{ml} \mathrm{m}^{-2}\right)$ & $39.7 \pm 7.2$ & $39.5 \pm 7.1$ & $42.8 \pm 7.8$ & $40.7 \pm 7.4^{*}$ \\
\hline $\operatorname{TFC}(k \Omega)$ & $29.2 \pm 4.1$ & $28.7 \pm 3.9$ & $31.9 \pm 4.8$ & $32.5 \pm 7.4$ \\
\hline $\mathrm{ACl}\left(/ 100 \mathrm{~s}^{2}\right)$ & $51.3 \pm 16.1$ & $47.6 \pm 21.5$ & $58.1 \pm 17.7$ & $51.4 \pm 12.9^{*}$ \\
\hline LCWI $\left(\mathrm{g} \mathrm{m}^{-1} \mathrm{~m}^{-2}\right)$ & $3.4 \pm 0.2$ & $3.4 \pm 0.8$ & $3.5 \pm 0.9$ & $3.4 \pm 0.9$ \\
\hline $\operatorname{PEP}(\mathrm{ms})$ & $85.0 \pm 24.9$ & $81.5 \pm 22.9$ & $82.7 \pm 21.7$ & $88.4 \pm 22.5$ \\
\hline LVET (ms) & $302.8 \pm 37.5$ & $306.0 \pm 35.5$ & $332.0 \pm 40.7$ & $319.3 \pm 30.7$ \\
\hline
\end{tabular}

Abbreviations: $\mathrm{ACl}$, acceleration index; BSA, body surface area; $\mathrm{Cl}$, cardiac index; CO, cardiac output; LCWI, left cardiac work index; LVET, left ventricular ejection time; PEP, pre-ejection period; SI, stroke volume index; STR, systolic time ratio; SV, stroke volume; SVR, systemic vascular resistance; SVRI, systemic vascular resistance index; TFC, thoracic fluid content; VI, velocity index. $\mathrm{SI}$, stroke volume index; STR, systolic time ratio; $\mathrm{S}$,
${ }^{P}<0.05$ when compared with the baseline values.

impaired coronary arterial blood supply, atherogenesis and/or microvascular damage. ${ }^{25}$ Here, we observed that LTP-treated patients experienced a significant improvement in vascular rigidity, as deduced from the increase in PWV. This result is partially in agreement with the recent observations of Japanese colleagues who used different instrumental markers of vascular rigidity. ${ }^{11-13}$ In fact, it seems that LTPs are able to improve the endothelial function independently from their effect on $\mathrm{BP}^{26}$ Therefore, this can at least partly explain our result of no significant relationship between SBP and PWV change. On the other hand, LTPs were also associated in our study with a mild but significant change in markers of cardiac flow (SV and stroke index) and of cardiac contractility (ACI and VI), but not in markers of fluid dynamics or vascular resistance. This could lead to a hypothesis of a mechanism of action different from angiotensin-converting enzyme inhibition, support for which would require further research. However, our observations on PWV suggest that the change in myocardial contractility could be a response of the heart to the reduced peripheral resistance. Therefore, to the best of our knowledge, there is actually no evidence of a direct effect of LTPs on myocardial contractility.

Our study had some limitations. First, the sample size of the study was relatively small, even if similar to most of the previous trials that investigated the antihypertensive effects of LTPs. Another limitation, also similar to previous trials, was the relatively short duration of the treatment, which could mask long-term treatment effects. Finally, the administration of a large number of tests to the patients on the same day could have influenced their spontaneous stress reactions and, consequently, their reactions to the measured BP. On the other hand, this is the first report of a complete set of data taken from LTP-treated patients, with respect to cardiac output-related parameters in particular.

In conclusion, VPP and IPP, assumed to be functional foods, positively influenced office SBP, PWV, SV, stroke index, ACI and VI in southern European patients with high-normal BP or first-degree hypertension.

\section{CONFLICT OF INTEREST}

The authors declare no conflict of interest.
1 Miller III ER, Erlinger TP, Appel LJ. The effects of macronutrients on blood pressure and lipids: an overview of the DASH and OmniHeart trials. Curr Atheroscler Rep 2006; 8: 460-465.

2 Pérez-López FR, Chedraui P, Haya J, Cuadros JL. Effects of the Mediterranean diet on longevity and age-related morbid conditions. Maturitas 2009; 64: 67-79.

3 Pripp AH. Effect of peptides derived from food proteins on blood pressure: a metaanalysis of randomized controlled trials. Food Nutr Res 2008; 52: 1-9.

4 Murray BA, FitzGerald RJ. Angiotensin converting enzyme inhibitory peptides derived from food proteins: biochemistry, bioactivity and production. Curr Pharm Des 2007; 13: 773-791.

5 FitzGerald RJ, Meisel H. Milk protein-derived peptide inhibitors of angiotensin-Iconverting enzyme. Br J Nutr 2000; 84 (Suppl 1): S33-S37.

6 Cicero AFG, Gerocarni B, Laghi L, Borghi C. Blood pressure lowering effect of lactotripeptides assumed as functional foods: a meta-analysis of current available clinical trials. J Hum Hypertens 2010; 25: 425-436.

7 Germino FW, Neutel J, Nonaka M, Hendler SS. The impact of lactotripeptides on blood pressure response in stage 1 and stage 2 hypertensives. J Clin Hypertens 2010; 12: 153-159.

8 Yamasue K, Morikawa N, Mizushima S, Tochikubo O. The blood pressure lowering effect of lactotoripeptides and salt intake in 24-h ambulatory blood pressure measurements. Clin Exp Hypertens 2010; 32: 214-220.

9 Cicero AFG, Rosticci M, Veronesi M, Bacchelli S, Strocchi E, Melegari C, Grandi E, Borghi C. Haemodynamic effects of lactotripeptides from casein hydrolysate in Mediterranean normotensive subjects and patients with high-normal blood pressure: a randomized, double-blind, cross-over clinical trial. J Med Food 2010; 13: 1363-1368.

10 van Mierlo LA, Koning MM, van der Zander K, Draijer R. Lactotripeptides do not lower ambulatory blood pressure in untreated whites: results from 2 controlled multicenter crossover studies. Am J Clin Nutr 2009; 89: 617-623.

11 Yoshizawa M, Maeda S, Miyaki A, Misono M, Choi Y, Shimojo N, Ajisaka R, Tanaka H. Additive beneficial effects of lactotripeptides intake with regular exercise on endothelium-dependent dilatation in postmenopausal women. Am J Hypertens 2010; 23: 368-372.

12 Yoshizawa M, Maeda S, Miyaki A, Misono M, Choi Y, Shimojo N, Ajisaka R, Tanaka H. Additive beneficial effects of lactotripeptides and aerobic exercise on arterial compliance in postmenopausal women. Am J Physiol Heart Circ Physiol 2009; 297: H1899-H1903.

13 Nakamura T, Mizutani J, Sasaki K, Yamamoto N, Takazawa K. Beneficial potential of casein hydrolysate containing Val-Pro-Pro and Ile-Pro-Pro on central blood pressure and hemodynamic index: a preliminary study. J Med Food 2009; 12: 1221-1226.

14 Mancia G, De Backer G, Dominiczak A, Cifkova R, Fagard R, Germano G, Grassi G, Heagerty AM, Kjeldsen SE, Laurent S, Narkiewicz K, Ruilope L, Rynkiewicz A, Schmieder RE, Boudier HA, Zanchetti A, ESH-ESC Task Force on the Management of Arterial Hypertension. ESH-ESC Practice Guidelines for the management of arterial hypertension: ESH-ESC Task Force on the management of arterial hypertension. J Hypertens 2007; 25: 1751-1762.

15 Pickering TG, Hall JE, Appel LJ, Falkner BE, Graves J, Hill MN, Jones DW, Kurtz T, Sheps SG, Roccella EJ. Recommendations for blood pressure measurement in humans and experimental animals: part 1: blood pressure measurement in humans: a statement for professionals from the Subcommittee of Professional and Public Education of the 
American Heart Association Council on High Blood Pressure Research. Circulation 2005; 111: 697-716.

16 Madin K, Iqbal P. Twenty four hour ambulatory blood pressure monitoring: a new tool for determining cardiovascular prognosis. Postgrad Med J 2006; 82: 548-551.

17 Monahan KD, Dyckman DJ, Ray CA. Effect of acute hyperlipidemia on autonomic and cardiovascular control in humans. J Appl Physiol 2007; 103: 162-169.

18 Tomiyama H, Yamashina A. Non-invasive vascular function tests: their pathophysiological background and clinical application. Circ J 2010; 74: 24-33.

19 Sodolski T, Kutarski A. Impedance cardiography: a valuable method of evaluating haemodynamic parameters. Cardiol J 2007; 14: 115-126.

20 Borghi C, Veronesi M, Cosentino E, Cicero AF, Kuria F, Dormi A, Ambrosioni E. Interaction between serum cholesterol levels and the renin-angiotensin system on the new onset of arterial hypertension in subjects with high-normal blood pressure. $J$ Hypertens 2007; 25: 2051-2057.

21 Wuerzner G, Peyrard S, Blanchard A, Lalanne F, Azizi M. The lactotripeptides isoleucine-proline-proline and valine-proline-proline do not inhibit the $\mathrm{N}$-terminal or
C-terminal angiotensin converting enzyme active sites in humans. J Hypertens 2009; 27: 1404-1409.

22 van der Zander K, Bots ML, Bak AA, Koning MM, de Leeuw PW. Enzymatically hydrolyzed lactotripeptides do not lower blood pressure in mildly hypertensive subjects. Am J Clin Nutr 2008; 88: 1697-1702.

23 Engberink MF, Schouten EG, Kok FJ, van Mierlo LA, Brouwer IA, Geleijnse JM. Lactotripeptides show no effect on human blood pressure: results from a doubleblind randomized controlled trial. Hypertension 2008; 51: 399-405.

24 Germino FW, Neutel J, Nonaka M, Hendler SS. The impact of lactotripeptides on blood pressure response in stage 1 and stage 2 hypertensives. J Clin Hypertens 2010; 12: 153-159.

25 Boutouyrie P, Laurent S, Briet M. Importance of arterial stiffness as cardiovascular risk factor for future development of new type of drugs. Fundam Clin Pharmacol 2008; 22: 241-246.

26 Hirota T, Ohki K, Kawagishi R, Kajimoto Y, Mizuno S, Nakamura Y, Kitakaze M. Casein hydrolysate containing the antihypertensive tripeptides Val-Pro-Pro and Ile-Pro-Pro improves vascular endothelial function independent of blood pressure-lowering effects: contribution of the inhibitory action of angiotensin-converting enzyme. Hypertens Res 2007; 30: 489-496. 